\title{
PROYECTO DE EXTENSIÓN: ENSEÑANZA DE ELE Y LA MOTIVACIÓN COMO HERRAMIENTAS EN LA FORMACIÓN CIUDADANA DEL ALUMNO EN LA AMAZONIA $^{1}$
}

\author{
Rita de Cássia PAIVA ${ }^{2}$ \\ Universidade Federal do Pará \\ Brenna Evelyn SOARES PEREIRA ${ }^{3}$ \\ Universidade Federal do Pará \\ Jacqueline Augusta LEITE DE LIMA ${ }^{4}$ \\ Universidade Federal do Pará
}

\begin{abstract}
RESUMO: Este trabalho dedica-se a demostrar como o ensino do espanhol como língua estrangera (E/LE) e o professor reflexivo e motivador são as principais ferramentas para a formação cidadã de alunos do projeto de ensino, pesquisa e extensão Guamá Bilíngue no bairro do Guamá que é uma das maiores periferias na Amazônia. Desde o ano 2010, este projeto utiliza o ensino do espanhol como cenário para levar as aulas textos sobre violência sexual, bullying, alcoolismo na adolescência, entre outros temas que fazem parte da vida cotidiana dos alunos, que tem de 15-18 anos e estão em risco social. Estes materiais servem para auxiliar a debater, refletir e instruir o jovem sobre os conteúdos diversos e fazer com que os estudantes da graduação de letras/espanhol que atuam no projeto se tornem professores reflexivos y motivadores, capazes de direcionar seus alunos a uma formação cidadã crítica para que atuem de maneira a contribuir positivamente na sociedade. Para tanto, foram utilizados os princípios de modelo mental (Lawson \& Lawson, 1993), as teorias de aprendizagem significativo (Ausubel apud Moreira \& Mansini, 1982) e premissas de Vygotsky (1991), entre outros. O Guamá Bilíngue agrega a função de facilitar uma relação transformadora entre a universidade e a sociedade que a cerca, e para este fim, objetiva principalmente a diminuição da violência e o preconceito social por meio do ensino de E/LE, motivando os alunos participantes a terem uma perspectiva de um futuro melhor. Dentro do mesmo afã, propõem que os alunos da graduação que participam do projeto sejam mais bem instruídos, treinados e motivados a atuar como docentes que motivem e transformem seu entorno, influenciando não somente na formação de seus próprios discentes como também no contexto en que estão inseridos.
\end{abstract}

PALAVRA-CHAVE: Formação docente; Guamá Bilíngue; Ensino de E/LE.

RESUMEN: Nos dedicamos aquí a demostrar como la enseñanza del español como lengua extranjera (ELE) y el profesor reflexivo y motivador son las principales herramientas para la formación ciudadana de alumnos del proyecto de enseñanza, investigación y extensión Guamá Bilingüe en el barrio del Guamá que es una de las mayores periferias en la Amazonia. Desde el año 2010, este proyecto utiliza la enseñanza del español como escenario para llevar a las clases textos sobre violencia sexual, acoso, alcoholismo en la adolescencia entre otros temas que hacen parte de la vida cotidiana de los alumnos, que tienen de 15-18 años y están

\footnotetext{
${ }^{1}$ Trabajo elaborado bajo investigaciones diversas del grupo Guamá Bilingüe: http://dgp.cnpq.br/dgp/espelhogrupo/5561497054806008

${ }^{2}$ Formación doctoral en Estudios de la Traducción en la UFSC; profesora coordinadora del curso de Letras Español, en la Universidad Federal de Pará. Contacto: cassia@ufpa.br

${ }^{3}$ Estudiante del último semestre de la carrera Letras Español en la Universidad Federal de Pará. Contacto: brennaevelyn@outlook.com.br

${ }^{4}$ Estudiante del último semestre de la carrera Letras Español en la Universidad Federal de Pará. Contacto: jacquelineleitedelima@gmail.com
} 
en riesgo social. Estos materiales sirven para auxiliar a debatir, reflexionar e instruir el joven acerca de contenidos diversos y hacer con que los estudiantes de la carrera de letras/español que actúan en el proyecto se conviertan en profesores reflexivos y motivadores capaces de direccionar sus alumnos hacia una formación ciudadana crítica para que actúen de manera a contribuir positivamente en la sociedad. Para tanto, fueron utilizados los principios de modelado mental (Lawson \& Lawson, 1993), las teorías del aprendizaje significativo (Ausubel apud Moreira \& Mansini, 1982) y premisas de Vygotsky (1991), entre otros. El Guamá Bilingüe agrega la función de facilitar una relación transformadora entre la universidad y la sociedad que la acoge, y para este fin, objetiva principalmente la disminución de la violencia y el prejuicio social por medio de la enseñanza de ELE motivando los alumnos participantes hacia una perspectiva de un mejor porvenir. En el mismo afán, propone que los alumnos de la carrera que se ocupan del proyecto sean más bien instruidos, entrenados y motivados a actuar como docentes que motiven y transformen su entorno, influyendo no solamente en la formación de sus propios discentes como también en el contexto en que estén insertados.

Palabras-clave: Formación docente, Guamá Bilingüe, Enseñanza de ELE

\section{Introducción}

El Siglo XXI ya ha aportado innúmeras novedades en el ámbito de la ciencia y tecnología. Las pizarras digitales, los ambientes simulados y el aula interactiva ya hacen parte del cotidiano de muchas instituciones educativas alrededor del globo. Saber manejar todo eso es considerado parte integrante de la formación del docente contemporáneo, fruto de todo un sistema comprometido con el futuro. Sin embargo, en la formación docente, sobre todo de español, en la región de la Amazonía brasileña, esto no es necesariamente verdad. Para intentar disminuir la distancia entre norte y sur, los aportes didáctico/pedagógicos pasan a ser la principal ventaja en la formación docente. Así, a través de un proyecto de investigación, enseñanza y extensión, el Guamá Bilingüe, se pretende dar herramientas a los alumnos para la formación ciudadana.

El proyecto Guamá Bilingüe, existe desde el 2010 y es fruto de una acción de la Pro Rectoría de Enseñanza y Graduación de la Universidad Federal de Pará -UFPA, en la amazonia brasileña, a través del PAPIM -Programa de Apoyo a Proyectos de Intervención Metodológica- que busca incentivar y apoyar el desarrollo de actividades y experimentos que acrecienten métodos y técnicas innovadoras y eficaces en el proceso de enseñanza y aprendizaje. En él, se congrega la oportunidad de los alumnos de la carrera Letras/Español de actuar como becarios o voluntarios de impartir clase a alumnos en riesgo social del barrio Guamá, donde se ubica la UFPA:

La UFPA está insertada en dos tercios dentro del barrio Guamá que es una zona de peligro para los residentes y más para los jóvenes. En el texto del proyecto se menciona que:

Este barrio es, reconocidamente, uno de los más violentos de esta capital debido a las desigualdades sociales y a los parcos recursos en él invertidos por los sectores públicos y privados para la mitigación de esta situación.

Siendo la UFPA una institución pública con una fuerte inclinación social en sus diversos cursos, está inserta en una relación de amensalismo ${ }^{5}$ con su entorno que le impide alcanzar su plenitud académica.

\footnotetext{
${ }^{5}$ Interacción biológica que se produce cuando un organismo se ve perjudicado en la relación y el otro no experimenta ninguna alteración.
} 
Partiendo de esa premisa, creemos que la educación -como agente transformador- puede iniciar un ciclo de cambios positivos que culminen en una relación más productiva para ambos, a saber, UFPA y barrio del Guamá. (GUAMÁ BILINGÜE, 2010)

\section{La formación del formador: Como el proyecto motiva el motivador.}

Pese que el proyecto Guamá Bilingüe posee una metodología de enseñanza de E/LE distinta, ya que su naturaleza está volcada a la enseñanza y practica social, pues la clasificamos, según GOHN (2010), como una educación no formal, que es basada en una vía de dos manos, es decir, que a la misma hora que el profesor aprende, el mismo también enseña. Creyendo en esto, el proyecto propone, en el mismo afán, incentivar la motivación en los alumnos que se benefician del proyecto, el alumno de la carrera de letras/español, tal como el alumno joven en riesgo social.

En este apartado trataremos de como trabajada el proyecto Guama Bilingüe en su mirada hacia el futuro profesor, pues creemos que para que el proyecto pueda intervenir sobre el alumno joven que se encuentra en riesgo social proporcionándole un cambio de pensamiento y conducta, es necesario que el interventor más directo, el profesor, sea efectivo, ya que es quien estará en clase llevando la dinámica del proyecto, debatiendo los temas propuestos y creando un vínculo con el alumno. Así es necesario que sea portador de estos ideales y comportamientos propuestos para la clase.

Sería perfecto si todas las personas, a lo mejor, todos los alumnos que ingresan en la carrera docente fueran ciudadanos totalmente conscientes de las conductas éticas de una sociedad, de lo que es correcto o no, de los prejuicios existentes en una comunidad y dentro de uno mismo, o que supieran las leyes de memoria. Pero no lo es así, algunos alumnos tienen ganas de actuar en una clase del proyecto Guamá Bilingüe e impartir una clase sobre el tema del acoso sin darse cuenta de que ha sido o sigue teniendo conductas de un acosador, o quizás para hablar de respetos y derechos en relaciones entre iguales pero produce un discurso homofóbico, y eso sin darse cuenta.

Así que antes de cualquier actuación que el alumno de la carrera pueda ejercer dentro de una clase del proyecto, él pasa por un proceso gradual de desconstrucción y formación docente y social. Los mismos temas propuestos a debatirse en clase son previamente debatidos entre la comisión técnico-pedagógica y los alumnos que participan del grupo de investigación como voluntarios, becarios o colaboradores, con el diferencial del debate está en la amplitud del tema. Cuando ellos son puestos para discusión, en las reuniones con los alumnos de la carrera, se desarrollan más al fondo haciendo enlaces con diversos temas relacionados.

Esta desconstrucción de algunos argumentos, como prejuicios, intolerancia, ética e imparcialidad religiosa, hecha sobre el alumno de la carrera es una de las manera que el proyecto posee de garantizar que el profesor que entre en el aula de Guamá Bilingüe consiga ser coherente con las ideologías que transmite al alumno, y sepa actuar siguiendo la propuesta del proyecto que va más allá que enseñar español, pues estamos de acordó con Freire (2012) y creemos que un profesor debe ser el ejemplo, de sus palabras.

Por eso, el proyecto posee en su metodología actividades que buscan influir positivamente en esta formación social y docente del futuro profesor del proyecto, las principales actividades 
para los participantes de la carrera de letras/español en el grupo son participar de las reuniones pedagógicas y de investigación, observar y auxiliar los profesores en el aula y la producción obligatoria de informes sobre el desarrollo de las clases.

Las reuniones pedagógicas son realizadas a cada quince días y destinadas a explicar, constantemente, la metodología e ideología del proyecto, direccionadas principalmente para los alumnos que tienen pretensión o ya actúan dentro del aula del proyecto, las reuniones pedagógicas buscan debatir los temas que serán trabajados en clase, hacer los alumnos reflexionar acerca de su propia opinión sobre estos temas, compartir o sugerir maneras de actuar en clase, presentar los problemas ocurridos en la actuación docente y buscar o proponer soluciones para los mismo, compartir relatos de sus experiencias en clases y dialogar con el grupo. O sea, en las reuniones pedagógicas es el espacio que el alumno tiene para en conjunto reflexionar, debatir y obtener una visión más amplia de sus pensamientos y conductas sociales y profesionales.

Las reuniones de investigación son realizadas quincenalmente y destinadas a tratar de los compromisos y actividades académicas de los participantes, como formulación de artículos, proposición de ponencias y discusión de la parte teórica de los estudios del proyecto. Los profesores del proyecto son incitados a actuar en clase y ser investigadores, sin embargo, de la misma manera que la formación docente es gradual, la producción académica también es gradual, utilizando los principios de andamiaje, según Vygotsky (1989), o sea un alumno con más experiencia académica o un participante del grupo con mayor nivel de grado auxilia el alumno menos experimentado a producir trabajos académicos sobre el proyecto en diversas áreas, creando una base de confianza en el alumno hasta que el mismo se sienta motivado y seguro alcanzar nuevos niveles profesionales.

Observar y auxiliar los profesores que ya actúan en clase no es una actividad obligatoria para los participantes del proyecto, sin embargo es uno de los requisitos que auxilia en la selección de los futuros profesores que actuaran en el proyecto, pues creemos que en esta actividad ocurre el proceso de aprendizaje por observación de Baldwin (1973), que según la definición de Borges-Andrade (1981), es la totalidad del proceso en donde el profesor actuante en clase expone el comportamiento y el observador es capaz de producir un aprendizaje imitativo repitiendo, cuando juzgado positivo, o rechazando un comportamiento, cuando juzgado inadecuando. Este juzgamiento hecho por el alumno que observa es expuesto en las reuniones y evaluado colectivamente para que el profesor y los demás compañeros puedan reflexionar acerca de la actitud tomada en clase.

Otra manera que el proyecto propone para que el alumno pueda reflexionar sobre su actuación en clase es la producción, obligatoria, de los informes del desarrollo de la clase que deben ser hechos a cada clase relatando todo el ocurrido, desde la planificación previa de la clase, el desarrollar de la misma, las peculiaridades en el debate con los alumnos, incluso lo que no salió como planteado y los problemas ocurridos. Estos informes son enviados a la comisión pedagógica, que los lee y selecciona los tópicos que deben ser comentados en las reuniones con la intención de hacer una reflexión acerca de la actuación de los profesores entrenándolos para ser profesionales reflexivos, según Schön (1987), a través de la práctica reflexiva.

Son estas actividades practicadas dentro del proyecto que forman el profesor del proyecto, capaz de reflexionar sobre la clase y lo que hay más allá del contenido, consciente de las conductas éticas y sociales a las cuales debe ser coherente al traspasárselas a los alumnos, no solamente en su discurso pero también siendo un ejemplo en clase para sus alumnos en termos sociales y linguísticos, además de tornarlos receptivos a críticas constructivas a través de la 
práctica reflexiva y motivado a crear una relación afectiva positiva con el alumno para que el objetivo del proyecto sea atingido y el joven sufra un cambio en su formación ciudadana

Además de esto, al final de la participación en el proyecto el alumno, principalmente los que actúan como profesores dentro del Guamá Bilingüe, se muestran más confiados en su nivel de lengua y en sus conductas docentes y más motivadas en su formación profesional debido a las experiencias vividas dentro del proyecto, incluso muchos alumnos de la carrera de letras/español al concluir la carrera siguen con su vínculo al proyecto, sea el académico, docente o afectivo, la mayoría se convierte en parte de la comisión técnico-pedagógica.

De esta manera, el alumno de la carrera llega a la clase como un profesional motivado y consciente de su ciudadanía, capaz de reflejar en el alumno su imagen y trabajar, según Freire (2012), una práctica testimonial, que es aplicar en sus alumnos bajo la experiencia semejante vivida por él, visto que el ejemplo refuerza la enseñanza social con el intuito de motivar el alumno a través del discurso del profesor en lugar de desdecirlo.

\section{La motivación en clases de ELE para alumnos en riesgo social.}

El proyecto Guamá Bilingüe y los demás proyectos oriundos del mismo, poseen una metodología que el enfoque es la formación ciudadana del alumno que se encuentra en zonas de vulnerabilidad social. Para alcanzar estos jóvenes, disponemos además de la formación de los profesores becarios que es volcada a formar una visión crítica y reflexiva sobre la vida del propio estudiante y la sociedad que él habita, un material didáctico que fue hecho con temas variados, conteniendo tanto temas culturales como sociales.

Este material posee temas que hablan de alcoholismo, igualdad de géneros, prejuicios, derechos humanos, acoso entre otros. Todos los temas son actuales y de gran relevancia. Por eso, en el momento que los profesores presentan los textos y actividades a ellos, despierta la voluntad del alumno de compartir con los otros sus vivencias, el papel del profesor es ser el mediador de la interacción entre ellos, debido a su formación anterior, él sabe cómo conducir esta reflexión, así los textos y actividades se tornan un punto de partida al dialogo haciendo del español la lengua vehicular.

Entendemos que "O fator isolado mais importante que influencia o aprendizado é aquilo que o aprendiz já conhece". Ausubel (1982) y este conocimiento previo del estudiante es uno de los factores que influyen en la motivación del estudiante para permanecer en el curso y como ocurre en muchos momentos, volver en los años siguientes para hacer el curso de lengua española nuevamente.

Y en esta cuestión, atentamos que el alumno se siente acogido en las clases, porque puede expresarse y sabe que en el proyecto sus opiniones, experiencia de vida y conocimientos de cotidiano serán respetadas, discutidas con los demás, valoradas y a veces reformuladas a través de este dialogo.

Sumado a este material con temas pertenecientes al cotidiano, consideramos que "De entre las lenguas románicas el portugués y el español son las que mantienen mayor afinidad entre sí" Almeida Filho (199, p. 14) así podemos dar clases de español halando en la lengua desde los primeros días, ellos aprenden mediante a lo que Ausubel (1982) llama de "Assimilação de conceitos" que define: 
A assimilação de conceitos é, caracteristicamente, a forma pela qual as crianças mais velhas, bem como os adultos adquirem novos conceitos pela recepção de seus atributos criteriais e pelo relacionamento desses atributos com ideias relevantes já estabelecidas em sua estrutura cognitiva.

Hay una gran similitud entre la lengua española y portuguesa, Ulsh (1971), (citado en Almeida Filho, (1995, p.14) establece que “[...] Más del 85\% del vocabulario portugués tiene cognados en español". Facilitando así, la comunicación en clase, la posibilidad de intercambio de ideas entre ellos, usando de inmediato la lengua española.

En este ánimo, tomamos a Méier (1974 citado en Paiva, 2008, p. 3284) ilustra que:

La adquisición de otra lengua implica la instalación de un nuevo código lingüístico, un nuevo sistema de codificación y decodificación, independiente del sistema utilizado hasta entonces. Podemos imaginar que esos dos sistemas se desarrollen o independientemente uno del otro, si la realidad cubierta por los significados es diferente, o en superposición, si los significados son interpretados sucesivamente en las dos lenguas.

Con la similitud entre el español y el portugués, diversas actividades pueden ser aplicadas confiando en que el propio pensamiento o insight analógico van a contribuir para el aprendizaje.

En lo que se refiere al desafío de hacer con que el ambiente de enseñanza de la lengua española sea motivador y contribuya a la formación ciudadana, es necesario comentar que hacer actividades divertidas, sin un compromiso a posibilitar la reflexión, la negociación de significados y llevar en consideración la investigación de los saberes de los alumnos, puede incapacitar una posible conexión en el aprendizaje.

Los factores determinantes para que el aprendizaje sea significativo en definitivo, es pensar que la compresión del asunto se da en la abstracción del estudiante. El aprendizaje significativo concebida por Ausubel es compatible con el concepto sociointeracionista de Vygotsky (1896-1934). Que considera que una persona necesita interactuar con otras, afín de que hay un desarrollo cognitivo, en otras palabras, el ambiente sociocultural, aunque, fabricado al estudiante, como en el caso del proyecto Guamá Bilingüe, cumple un papel importante en la formación ciudadana.

Sabemos que cada alumno se motiva por razones distintas, al profesor se queda la tarea de ser un agente exterior que trata en reflexionar sobre su práctica en clase, para que posteriormente, pueda aplicar de la forma más motivadora posible. Es importante que el profesor note, que una misma actividad incentivadora produce distintas respuestas en distintos individuos, o incluso el estudiante mismo en diversos momentos.

Es importante que el Profesor becario se apoye en los intereses de los alumnos y consiga conectarlos con los objetivos del tema propuesto para reflexión o con la actividad. Conforme sugiere el modelado mental de Lawson \& Lawson (1993) a través de las analogías hechas entre la vida del alumno e el tema abordado.

Se puede tomar por ejemplo, la primera unidad del material didáctico que muestra el texto de dos ex alumnas de un proyecto anterior que contemplaba los mismos valores del Guamá Bilingüe: 

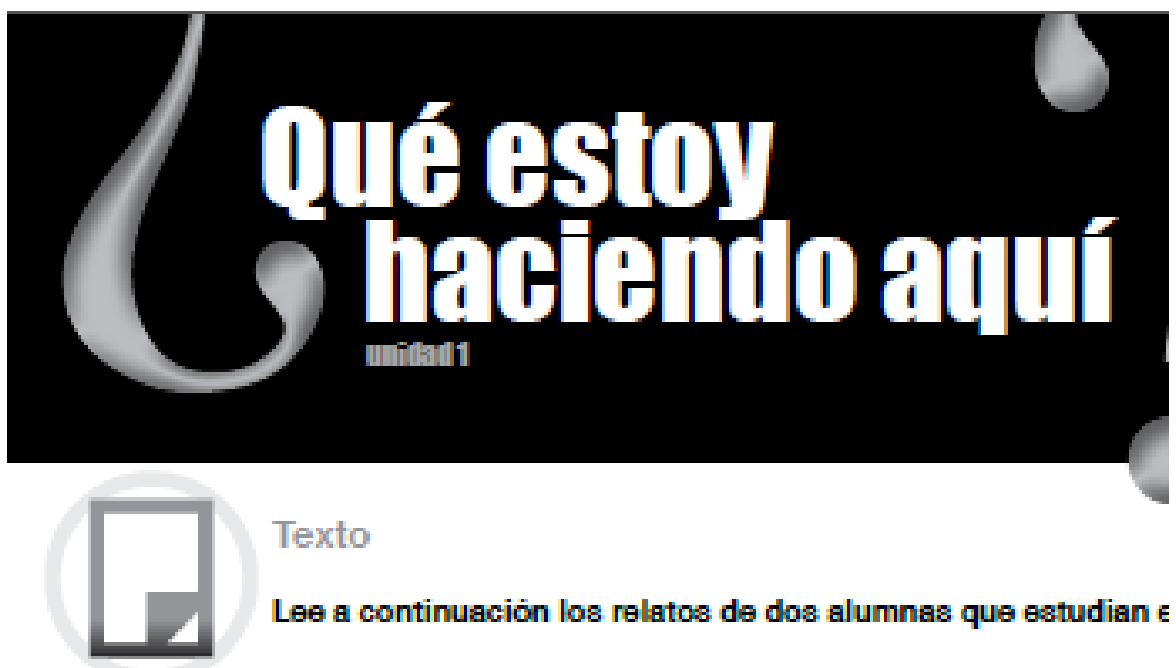

Texto

Lee a continuación los relatos de dos alumnas que estudian $\epsilon$

Relato I

Escrtoo para cortar en que el espanol mejoro mi wha

SI no me equivoon, comenob a hacer curso de expafol 0013 afos en un proyocto sodil. Conficesof

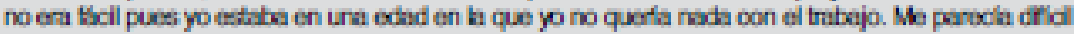
omntus para estudar y dejar de divertime los seltwodos por bs tarbes para aprender otra kengua que squera sabla sd irfa a uar...

Pero valio la pera. Las cpotunibades vinieron. Hoy lengo 21 aflos ye vajé a un pals hizanohablart trabajo en una empresa inareracional. Puedo docir que solo conexgul eritrar en esta empresa porqua expafol. Hoy elbe también me ockran al ingés.

Digo a todos que, por mats offel que sae aprender, por más que no iengamos dinen, por mís beanen

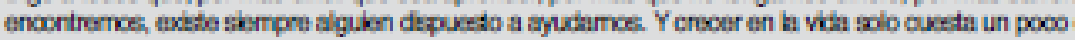
efferzo y varias renunches.

Nanuzes,

Parmela Pacheoo

Rielato II

¡Hola nuevos alumncel

Mi nombre es Kattia Diniz. Estudib expafol en un proyecto sockal hace abunce afos y lo necesto tr dias porque vivo en la Cudad de Mexico desde 2006 .

Vne a trabajar por tres meoss y todwala sigo aqui Soy modelo y pate de mi trabajo es hablar, tanta cliertes como frerte a b camara, lo que es mucho más difici.

E idibera refleja el modo de persar y de vivir de los dferentes puctice. Es el primer peso para ente dfecentes culturas y parn adaptanse a un lugar bjos de casa. Ededen oceas que son obvias para u muy libgicas o hexplicabies para otroe. Caando sales de casa, no expenas que dilerenciss tan chiq puedan afectarte como, por ejemplo, que te informen una divoción inconecta, que no se coda ef la una sefora mayor en el metro o, incluso, que en la comida no vengan jurtice el arroz y el trijot.

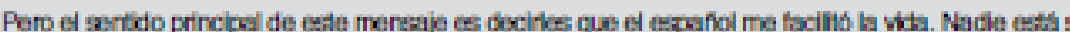

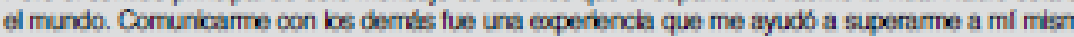
comprender has dfferenchas, entender abeas que antes no entendla y cambiar mi vida de una forme nunca habla imsginada. Sin el espanol no podifa haoer mi trabajo pues ahom ya vivo en Méxion he afos y medo. M próximo reto es hablar espafol shacerto.

Saludos y den bo mejor de si miemos sikmpre.

Figura I

Otro ejemplo es uno de los temas más incómodos que hace con que los alumnos del proyecto reaccionen a cosas de su cotidiano: 


\section{Aleoholismo adolosente

\section{El alcohol daña el cerebro adolescente}

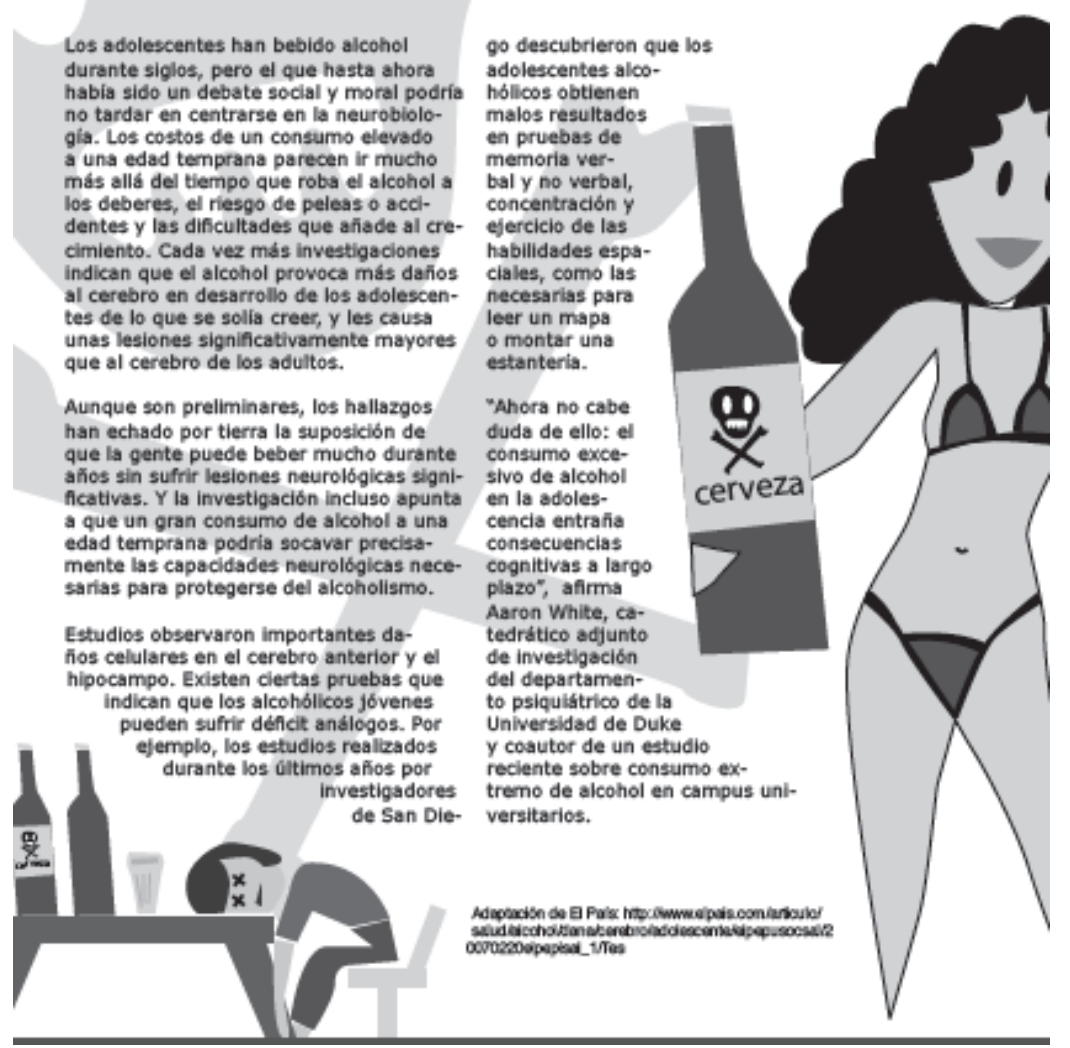

Figura 2

Temas como estos hacen parte de la formación del futuro docente que participa del proyecto. Sabemos que hay muchos profesores que buscan estrategias variadas, pero que a veces no provocan ninguna motivación en los alumnos. Los alumnos no se motivan de la misma manera por eso el profesor debe estar atento a su clase y buscar realizar actividades motivadoras que impliquen mayor participación del alumno, como la exposición de ideas.

Procesos permanecen siempre y sirven de refuerzo o motivación para posteriores aprendizajes. Esta manera de exponer sus ideas, mientras aprende español y el conjunto de es cargada con posibilidades de éxito, y el éxito anima al aprendizaje, conforme la participación el alumno se da cuenta de la utilidad de la lengua y de los diálogos, mientras, se entretiene y divierte.

El clima relacional de la clase, el ánimo del profesor y las buenas relaciones entre alumnos y profesor, el gusto por ser actuante en la clase y conseguir relacionar lo que se está aprendiendo con el mundo real, los hechos y experiencias del alumno, todo forma parte de la enseñanza de español para formación ciudadana. 


\section{Consideraciones Finales}

El Guamá Bilingüe, y ahora sus dos renuevos, agrega la función de facilitar una relación transformadora entre la universidad y la sociedad que la acoge, y para este fin, objetiva principalmente la disminución de la violencia y el prejuicio social por medio de la enseñanza de ELE motivando los alumnos participantes hacia una perspectiva de un mejor porvenir. En el mismo afán, propone que los alumnos de la carrera que se ocupan de los proyectos sean más bien instruidos, entrenados y motivados a actuar como docentes que motiven y transformen su entorno, influyendo no solamente en la formación de sus propios discentes sino también en el contexto en que estén insertados.

\section{Referencias}

BALDWIN, A.L. Social Learning. Em F.N. Kerlinger (Ed.) Review of research in education (Vol. 1). Itasca. I11.: F.E. Peacock Publishers, 1973, 34-57. In: BORGES-ANDRADE, J. E. Aprendizagem por observação: perspectivas teóricas e contribuições para o planejamento instrucional - uma revisão. Psicol. cienc. prof., Brasília , v. 1, n. 2, p. 2-68, jul. 1981. Disponíble en <http://pepsic.bvsalud.org/scielo.php?script=sci_arttext\&pid=S1414$98931981000200001 \& \operatorname{lng}=$ pt\&nrm=iso $>$. Accedido en 04 de diciembre de 2016.

FREIRE, P. Pedagogía de la autonomía. Buenos Aires: Siglo Veintiuno, 2012.

GOHN, M. da G. Educação não formal e o educador social. São Paulo: Cortez, 2010.

GUAMÁ BILINGÜE. Disponible en: http://www.ufpa.br/beiradorio/novo/index.php/2010/116-edicao-86setembro/1084-guamabilingue-em-10-anos http://www.sisprol.ufpa.br/view/projeto/index.php?view=projeto\&action=consultarStatus Accedido en 26 de diciembre de 2011 a las 11:25.

PAIVA, R. C. O espanhol no ensino cotidiano. In: I Congresso Internacional Da Associação Brasileira de Hispanistas e V Congresso Brasileiro de Hispanistas, Belo Horizonte. Anais... Belo Horizonte: UFMG, 2008 p. 3282-3291.

SCHÖN, D. La formación de profesionales reflexivos. Hacia un nuevo diseño de la enseñanza y el aprendizaje en las profesiones. Editorial Paidós, Barcelona. 1992.

VYGOTSKY, L.S. Pensamento e Linguagem. São Paulo: Martins Fontes, 1989.

ALMEIDA FILHO, J. C. P. Uma metodologia específica para o ensino de línguas próximas? En JOSÉ CARLOS PAES DE ALMEIDA FILHO (org), Português para Estrangeiros: Interface com o Espanhol. Pontes, Campinas, SP,1995.

MOREIRA, M.; MASINI, E. F. S. Aprendizagem significativa: A teoria de David Ausubel. São Paulo: Moraes, 1982. 
LAWSON D. I. \& LAWSON A. E. Neural Principles of Memory and a Neural Theory of Analogical Insight. Journal of Research in Science Teaching, v30 n10 p1327-48/Dec 1993. 